\title{
An Extremal Property of Turán Graphs
}

\author{
Felix Lazebnik* Spencer Tofts \\ Department of Mathematical Sciences \\ University of Delaware, Newark, DE 19716, USA \\ lazebnik@math.udel.edu tofts@udel.edu \\ Submitted: Jul 6, 2010; Accepted: Nov 19, 2010; Published: Dec 10, 2010 \\ Mathematics Subject Classification: 05C15, 05C30, 05C31, 05C35
}

\begin{abstract}
Let $\mathcal{F}_{n, t_{r}(n)}$ denote the family of all graphs on $n$ vertices and $t_{r}(n)$ edges, where $t_{r}(n)$ is the number of edges in the Turán's graph $T_{r}(n)$ - the complete $r$-partite graph on $n$ vertices with partition sizes as equal as possible. For a graph $G$ and a positive integer $\lambda$, let $P_{G}(\lambda)$ denote the number of proper vertex colorings of $G$ with at most $\lambda$ colors, and let $f\left(n, t_{r}(n), \lambda\right)=\max \left\{P_{G}(\lambda): G \in \mathcal{F}_{n, t_{r}(n)}\right\}$. We prove that for all $n \geq r \geq 2, f\left(n, t_{r}(n), r+1\right)=P_{T_{r}(n)}(r+1)$ and that $T_{r}(n)$ is the only extremal graph.
\end{abstract}

\section{Introduction}

All graphs in this paper are finite, undirected, and have neither loops nor multiple edges. For all missing definitions and facts which are mentioned but not proved, we refer the reader to Bollobás [3].

For a graph $G$, let $V(G)$ and $E(G)$ denote the vertex set of $G$ and the edge set of $G$, respectively. Let $|A|$ denote the cardinality of a set $A$. Let $n=v(G)=|V(G)|$ and $m=e(G)=|E(G)|$ denote the number of vertices the (order) of $G$, and number of edges the (size) of $G$, respectively. An edge $\{x, y\}$ of $G$ will also be denoted by $x y$, or $y x$. For sets $X, Y$, let $X-Y=X \backslash Y$. For $A \subseteq V(G)$, let $G[A]$ denote the subgraph of $G$ induced by $A$, which means that $V(G[A])=A$, and $E(G[A])$ consists of all edges $x y$ of $G$ with both $x$ and $y$ in $A$. For a vertex $v$ of $G$, let $N(v)=N_{G}(v)=\{u \in V(G): u v \in E(G)\}$ denote the neighborhood of $v$ in $G$, and $d(v)=d_{G}(v)=\left|N_{G}(v)\right|$ denote the degree of $v$ in $G$. For $A \subseteq V(G)$, let $d_{A}(v)=\left|A \cap N_{G}(v)\right|$ denote the number of neighbors of a vertex $v$ in $G$ which are in $A$. For two disjoint nonempty subsets $A, B \subseteq V(G)$, by $G[A, B]$

*This research was partially supported by the NSA, Grant H98230-08-1-0041. 
we denote the bipartite subgraph of $G$ such that $V(G[A, B])=A \cup B$, and $E(G[A, B])$ consists of all edges of $G$ with one end-vertex in $A$ and the other in $B$.

A partition of a set $S$ is a collection of its disjoint nonempty subsets, $A_{1}, A_{2}, \ldots, A_{k}$, such that $S=A_{1} \cup A_{2} \cup \ldots \cup A_{k}$. A graph $G$ is called $r$-partite, $r \geq 1$, with nonempty vertex classes $V_{1}, V_{2}, \ldots, V_{r}$ if $V(G)$ is the disjoint union of $V_{1}, V_{2}, \ldots, V_{r}$ and every edge connects two vertices in different vertex classes. We say $G$ is a complete $r$-partite graph if it is $r$-partite and every two vertices in different vertex classes are connected. If $r=2$, and the vertex classes have $a$ and $b$ vertices, the complete 2-partite graph has $a b$ edges, and it is usually denoted by $K_{a, b}$. For $r=n$, the complete $n$-partite graph of order $n$ is called the complete graph of order $n$; it is denoted by $K_{n}$, and it has all possible $\left(\begin{array}{l}n \\ 2\end{array}\right)$ edges. For $r=1$, the complete 1-partite graph or order $n$ has no edges, hence, it is $\frac{2}{K_{n}}$ (the complement of $K_{n}$ ). The Turán graph $T_{r}(n), r \geq 1$, is the complete $r$-partite graph of order $n$ with all parts of size either $\lfloor n / r\rfloor$ or $[n / r\rceil$. It is easy to argue that such a graph is unique. For example, if $r=1, T_{1}(n)=\overline{K_{n}}$. If $r=2, T_{2}(n)$ is $K_{a, a}$ for $n=2 a$, and $K_{a+1, a}$ for $n=2 a+1$. If $r=n, T_{n}(n)=K_{n}$. Let $t_{r}(n)=e\left(T_{r}(n)\right)$ denote the number of edges of $T_{r}(n)$.

For a positive integer $\lambda$, let $[\lambda]=\{1,2, \ldots, \lambda\}$. A function $c: V(G) \rightarrow[\lambda]$ such that $c(x) \neq c(y)$ for every edge $x y$ of $G$ is called a proper vertex coloring of $G$ in at most $\lambda$ colors, or simply a $\lambda$-coloring of $G$. The set $[\lambda]$ is often referred to as the set of colors.

Let $P_{G}(\lambda)$ denote the number of all $\lambda$-colorings of a given graph $G$. This number was introduced and studied by Birkhoff [2], who proved that it is always a polynomial in $\lambda$. It is now called the chromatic polynomial of $G$. Although $P_{G}(\lambda)$ has been studied for its own sake, perhaps more interestingly there is a long history of diverse applications which has led researchers to minimize or maximize $P_{G}(\lambda)$ over various families of graphs. A good source of related references can be found in a recent article by Loh, Pikhurko and Sudakov [8]. We would like to add to that list surveys by Read [10], Read and Tutte [11], Read and Royle [12], and recent preprints by Norine [9] and Zhao [16].

Let $\mathcal{F}_{n, m}$ consist of all $(n, m)$-graphs, that is, graphs of order $n$ and size $m$. The problem of minimizing $P_{G}(\lambda)$ over $\mathcal{F}_{n, m}$ was solved by Linial [7], who showed that for any $n, m$, there is a graph which simultaneously minimizes each $\left|P_{G}(\lambda)\right|$ over $\mathcal{F}_{n, m}$, for every integer $\lambda$. This graph is simply a clique $K_{t}$ with an additional vertex adjacent to $s$ vertices of the $K_{t}$, plus $n-t-1$ isolated vertices, where $t, s$ are the unique integers satisfying $m=\left(\begin{array}{l}t \\ 2\end{array}\right)+s$ with $0 \leq s<t$. At the end of his paper, Linial posed the problem of maximizing $P_{G}(\lambda)$ over all graphs in $\mathcal{F}_{n, m}$. The same maximization problem, was also considered at around the same time by Wilf (and his motivation was different). See Wilf [15], and Bender and Wilf [1]. The maximization problem turned out to be much more difficult, and was only solved in sporadic cases. Let

$$
f(n, m, \lambda)=\max \left\{P_{G}(\lambda): G \in \mathcal{F}_{n, m}\right\} .
$$


Here is a list of known "exact" results on $f(n, m, \lambda)$. Many various bounds on this function can be found in the aforementioned references.

- The value of $f(n, m, 2)$ was determined, and all extremal graphs were characterized for all $m, n$ in Lazebnik [4].

- In Lazebnik [5] it was proved that $f\left(n, t_{r}(n), \lambda\right)=P_{T_{r}(n)}(\lambda)$, and that $T_{r}(n)$ is the only extremal graph for all $r \geq 1$ and all large $\lambda=\Omega\left(n^{6}\right)$, as $n \rightarrow \infty$.

- In Lazebnik, Pikhurko and Woldar [6], it was shown that for all $t \geq 1, f\left(2 t, t^{2}, 3\right)=$ $P_{K_{t, t}}(3)$, and that $T_{2}(2 t)=K_{t, t}$ is the only extremal graph. Thus it extended the result from [5] to a small $\lambda$, namely $\lambda=3$, but to only bipartite Turán graphs $T_{2}(2 t)$. It was also shown in [6] that

$$
f\left(2 t, t^{2}, 4\right) \sim P_{T_{2}(2 t)}(4) \sim(6+o(1)) 4^{t}, \quad \text { as } t \rightarrow \infty .
$$

This can be stated in other words, as the graph $T_{2}(2 t)$ is asymptotically extremal for $\lambda=4$.

- Most recently, Loh, Pikhurko and Sudakov [8] proved that for every $r \geq 3$, there exists $n_{0}=n_{0}(r)$, such that for all $n \geq n_{0}$,

$$
f\left(n, t_{r}(n), r+1\right)=P_{T_{r}(n)}(r+1),
$$

and that $T_{r}(n)$ is the only extremal graph. This result extends the one on $f\left(2 t, t^{2}, 3\right)$ from [6] to all $r \geq 3$, but it holds only for sufficiently large $n$. Though an explicit lower bound on $n_{0}$ was not specified in [8], it must be super-exponential as $r \rightarrow \infty$, as the proof relies on a stability result of Simonovits, which, in turn, uses regularity. Among other interesting results in [8], is a proof for large $m$ of a conjecture from [4] concerning the value of $f(n, m, 3)$ and the structure of extremal graphs in the case when an $m \leq n^{2} / 4$. It stated that the extremal graphs are complete bipartite graphs with certain ratio of partition sizes minus a star plus some isolated vertices if necessary. In addition, it is shown in [8], that for all $\lambda \geq 4$, large $m$, and $m \approx \frac{1}{\lambda \log \lambda} n^{2}$, the structure of extremal graphs is similar to the case of 3-colorings.

- Recently Norine [9] showed that for any positive integers $r, \lambda$, such that $2 \leq r<\lambda$ and $r$ divides $\lambda$, there exists $n_{0}=n_{0}(r, \lambda)$, such that for all $n \geq n_{0}$,

$$
f\left(n, t_{r}(n), \lambda\right)=P_{T_{r}(n)}(\lambda)
$$

and that $T_{r}(n)$ is the only extremal graph.

We are now ready to present the main result of this paper.

Theorem 1. For all integers $n, r$, with $n \geq r \geq 1$,

$$
f\left(n, t_{r}(n), r+1\right)=P_{T_{r}(n)}(r+1),
$$

and $T_{r}(n)$ is the only extremal graph. 
In relation to the aforementioned results, Theorem 1 represents the following improvements.

- It establishes the equality $f\left(n, t_{r}(n), \lambda\right)=P_{T_{r}(n)}(\lambda)$ for all $n \geq r \geq 1$ and $\lambda=r+1$ (for $\lambda=r$ the statement is an immediate corollary of Turán's theorem, see Turán $[14])$. Previously it was known only for large $\lambda=\Omega\left(n^{6}\right)([5])$.

- It generalizes the equality $f\left(2 t, t^{2}, 3\right)=P_{T_{2}(2 t)}(3)$ in [6] to all $\left(n, t_{r}(n), r+1\right)$-graphs with $r \geq 2$, and it covers the missing special case for $r=2$ and $n=2 t+1$.

- It extends the equality $f\left(n, t_{r}(n), r+1\right)=P_{T_{r}(n)}(r+1)$ in [8] from 'sufficiently large' $n$ to all $n \geq r$.

The proof of Theorem 1, which first appeared in Tofts [13], is presented in Section 2. This proof grew out of our attempts to extend the aforementioned result on $f\left(2 k, k^{2}, 3\right)$ from $[6]$ to $f\left(3 k, 3 k^{2}, 4\right)$. After finally resolving this case and simplifying the method several times, we began seeing the light: a much simpler and more general argument. It represents a 'correct' generalization of the main idea behind Theorem 3 in [6].

\section{Proof of Theorem 1}

Part (i) of the following lemma gives an explicit expression for $P_{T_{r}(n)}(r+1)$, which is an essential tool in our proof of Theorem 1. Though it appears in the Appendix of [8], we present our proof of this simple fact (obtained independently) for the sake of completeness.

Lemma 2.1 Let $n$ and $r$ be positive integers, such that $1 \leq r \leq n$. Let $k=\left\lfloor\frac{n}{r}\right\rfloor \geq 1$, and let $s=n-r k, 0 \leq s<r$. Then

(i) $P_{T_{r}(n)}(r+1)=(r+1) !\left(s 2^{k}+(r-s) 2^{k-1}-(r-1)\right)$.

(ii) For $n \geq r+1, P_{T_{r}(n-1)}(r+1)<P_{T_{r}(n)}(r+1)$.

Proof of (i): Denote the maximal independent sets of $T_{r}(n)$ by $V_{1}, V_{2}, \ldots, V_{r}$, such that $\left|V_{1}\right|=\left|V_{2}\right|=\ldots=\left|V_{s}\right|=k+1$, and $\left|V_{s+1}\right|=\left|V_{s+2}\right|=\ldots=\left|V_{r}\right|=k$. Take a proper $(r+1)$-coloring of $T_{r}(n)$. It is clear that it must use at least $r$ colors, and that if it uses all $r+1$ colors that there exists exactly one $V_{i}$ whose points are colored using two colors. Therefore, in order to compute $P_{T_{r}(n)}(r+1)$, we consider three cases.

Case 1: Exactly $r$ colors are used.

Obviously, there are exactly $\left(\begin{array}{c}r+1 \\ r\end{array}\right) r !=(r+1)$ ! colorings in this case. 
Case 2: All $r+1$ colors are used, and there exists exactly one $i, 1 \leq i \leq s$, such that $V_{i}$ 's points are colored in two colors.

There are $s$ ways to choose such $V_{i}$, and there are $\left(\begin{array}{c}r+1 \\ 2\end{array}\right)$ ways to choose two colors for it. There are $2^{\left|V_{i}\right|}-2$ ordered partitions of $V_{i}$ into 2 subsets, therefore there are $2^{\left|V_{i}\right|}-2$ ways of coloring it with the chosen two colors. Finally, there are $(r-1)$ ! ways to color the remaining $(r-1) V_{j}$ 's with the remaining $(r-1)$ colors. So, there is a total of

$$
s \cdot\left(\begin{array}{c}
r+1 \\
2
\end{array}\right) \cdot\left(2^{\left|V_{i}\right|}-2\right) \cdot(r-1) !=s(r+1) !\left(2^{k}-1\right)
$$

colorings in this case.

Case 3: All $r+1$ colors are used, and there exists exactly one $i, s+1 \leq i \leq r$, such that $V_{i}$ 's points are colored in two colors.

There are $r-s$ ways to choose such $V_{i}$, and there are $\left(\begin{array}{c}r+1 \\ 2\end{array}\right)$ ways to choose two colors for it. There are $2^{\left|V_{i}\right|}-2$ ordered partitions of $V_{i}$ into 2 subsets, therefore there are $2^{\left|V_{i}\right|}-2$ ways of coloring it with the chosen two colors. Finally, there are $(r-1)$ ! ways to color the remaining $(r-1) V_{j}$ 's with the remaining $(r-1)$ colors. So, there is a total of

$$
(r-s) \cdot\left(\begin{array}{c}
r+1 \\
2
\end{array}\right) \cdot\left(2^{\left|V_{i}\right|}-2\right) \cdot(r-1) !=(r-s)(r+1) !\left(2^{k-1}-1\right)
$$

colorings in this case.

Therefore, we have a total of

$(r+1) !+s(r+1) !\left(2^{k}-1\right)+(r-s)(r+1) !\left(2^{k-1}-1\right)=(r+1) !\left(s 2^{k}+(r-s) 2^{k-1}-(r-1)\right)$

colorings, as desired.

Proof of (ii): Assume $s=0$. Then, $n=r k$, since $n \geq r+1, k \geq 2$, and $n-1=$ $r(k-1)+(r-1)$. Using part $(i)$, we obtain

$$
\begin{aligned}
P_{T_{r}(n)}(r+1)-P_{T_{r}(n-1)}(r+1) & =(r+1) !\left(r 2^{k-1}-(r-1)\right) \\
& -(r+1) !\left((r-1) 2^{k-1}+2^{k-2}-(r-1)\right) \\
& =(r+1) !\left(2^{k-1}-2^{k-2}\right) \\
& >0
\end{aligned}
$$

However, if $s \geq 1$, then by part $(i)$, we find 


$$
\begin{aligned}
P_{T_{r}(n)}(r+1)-P_{T_{r}(n-1)}(r+1) & =(r+1) !\left(s 2^{k}+(r-s) 2^{k-1}-(r-1)\right) \\
& -(r+1) !\left((s-1) 2^{k}+(r-s+1) 2^{k-1}-(r-1)\right) \\
& =(r+1) !\left(2^{k}-2^{k-1}\right) \\
& >0
\end{aligned}
$$

Therefore, $P_{T_{r}(n)}(r+1)>P_{T_{r}(n-1)}(r+1)$.

Having established Lemma 2.1, we are ready to prove Theorem 1.

Proof of Theorem 1. We will use induction on $n$. The $r=1$ case is trivial, and the $r=2$, $s=0$ case was proved in Theorem 1 in [6], so we assume that $r \geq 3$, or $r \geq 2$ and $s \geq 1$.

Now, if $n=r$, the result is obvious, as $T_{r}(n)=T_{n}(n)=K_{n}$ and this is the only $\left(n, t_{r}(n)\right)$ graph. Therefore, suppose the theorem is true for all $m$ such that $2 \leq r \leq m<$ $n=r k+s, k=\left\lfloor\frac{n}{r}\right\rfloor \geq 1,0 \leq s<r$.

Let $G$ be a $\left(n, t_{r}(n)\right)$ graph not isomorphic to $T_{r}(n)$. Then, by Turán's Theorem, $G$ contains a subgraph isomorphic to $K_{r+1}$. Let the set of vertices of this complete subgraph be $A=\left\{u_{1}, \ldots, u_{r+1}\right\}$. Our proof is divided into two cases, depending on whether the value of $\sum_{i=1}^{r+1} d\left(u_{i}\right)$ is less than $(r+1)((r-1) k+s)$, or at least $(r+1)((r-1) k+s)$, and the arguments used in each case will differ.

Case 1: We assume that

$$
d\left(u_{1}\right)+d\left(u_{2}\right)+\ldots+d\left(u_{r+1}\right) \leq(r+1)((r-1) k+s)-1 .
$$

We show that in this case at least one vertex $u_{i}$ has degree small enough that its deletion from $G$ results in a graph with more that $t_{r}(n-1)$ edges, and the proof of the theorem will easily follow. Let $u_{i}$ be a vertex in $A$ with the lowest degree. Then

$$
d\left(u_{i}\right) \leq \frac{1}{r+1} \sum_{j=1}^{r+1} d\left(u_{j}\right)<(r-1) k+s .
$$

Case 1.1: Suppose that $s \geq 1$. As $n=r k+s, V\left(T_{r}(n-1)\right)$ is partitioned into $s-1$ parts each having $k+1$ vertices and $r-(s-1)$ parts each having $k$ vertices. Therefore we have:

$$
t_{r}(n)=t_{r}(r k+s)=\left(\begin{array}{l}
s \\
2
\end{array}\right) \cdot(k+1)^{2}+s(r-s) \cdot(k+1) k+\left(\begin{array}{c}
r-s \\
2
\end{array}\right) \cdot k^{2},
$$

and

$$
t_{r}(n-1)=t_{r}(r k+(s-1))=
$$




$$
\left(\begin{array}{c}
s-1 \\
2
\end{array}\right) \cdot(k+1)^{2}+(s-1)(r-s+1) \cdot(k+1) k+\left(\begin{array}{c}
r-s+1 \\
2
\end{array}\right) \cdot k^{2} .
$$

Therefore

$$
t_{r}(n)-t_{r}(n-1)=(r-1) k+(s-1) \geq d\left(u_{i}\right) .
$$

Case 1.2: Suppose $s=0$. In this case, $V\left(T_{r}(n-1)\right)$ is partitioned into $r-1$ parts each having $k$ vertices and one part having $k-1$ vertices. So $n-1=r k-1=r(k-1)+(r-1)$, and we have:

$$
t_{r}(n)=\left(\begin{array}{l}
r \\
2
\end{array}\right) \cdot k^{2}
$$

and

$$
t_{r}(n-1)=\left(\begin{array}{c}
r-1 \\
2
\end{array}\right) \cdot k^{2}+\left(\begin{array}{c}
r-1 \\
1
\end{array}\right) \cdot(k-1) k
$$

Therefore

$$
t_{r}(n)-t_{r}(n-1)=(r-1) k>d\left(u_{i}\right) .
$$

Let $G^{\prime}=G\left[V(G)-\left\{u_{i}\right\}\right]$. Then $v\left(G^{\prime}\right)=n-1$ and $e\left(G^{\prime}\right)>t_{r}(n-1)$. Also, $G^{\prime}$ contains a copy of $K_{r}$, namely $G^{\prime}\left[A-\left\{u_{i}\right\}\right]$. As $u_{i}$ is adjacent to all its vertices, there exists at most one way to extend a proper $(r+1)$-coloring of $G^{\prime}$ to the one of $G$. Therefore, $P_{G}(r+1) \leq P_{G^{\prime}}(r+1)$. Deleting edges from $G^{\prime}$, we can obtain a graph $G^{\prime \prime}$ such that $v\left(G^{\prime \prime}\right)=n-1$ and $e\left(G^{\prime \prime}\right)=t_{r}(n-1)$. Then $P_{G^{\prime}}(r+1) \leq P_{G^{\prime \prime}}(r+1)$, and, as $n-1 \geq r$, we have $P_{G^{\prime \prime}}(r+1) \leq P_{T_{r}(n-1)}(r+1)$ by the induction hypothesis. Therefore, we have

$$
P_{G}(r+1) \leq P_{G^{\prime}}(r+1) \leq P_{G^{\prime \prime}}(r+1) \leq P_{T_{r}(n-1)}(r+1)<P_{T_{r}(n)}(r+1),
$$

where the last inequality follows from Lemma 2(ii). This ends the proof of Case 1.

Case 2: We assume that

$$
d\left(u_{1}\right)+d\left(u_{2}\right)+d\left(u_{3}\right)+\ldots+d\left(u_{r+1}\right) \geq(r+1)((r-1) k+s) .
$$

For each $i, 0 \leq i \leq r+1$, let us define the following subsets of $V(G)-A$ :

$$
B_{i}=\left\{v \in V(G)-A \mid d_{A}(v)=i\right\} .
$$

If $G$ contains a subgraph isomorphic to $K_{r+2}$, then $P_{G}(r+1)=0<P_{T_{r}(n)}(r+1)$, and the proof is finished. Therefore, we assume that $G$ contains no $(r+2)$-clique. Then $B_{r+1}=\emptyset$ and $V(G)$ is the union of $r+2$ pairwise disjoint subsets (with some possibly empty):

$$
V(G)=A \cup B_{0} \cup B_{1} \cup \ldots \cup B_{r} .
$$

Let $b_{i}=\left|B_{i}\right|$ for $i=0, \ldots, r$. Since $G[A]$ is an $(r+1)$-clique,

$$
e(G[A, V(G)-A])=d\left(u_{1}\right)+d\left(u_{2}\right)+d\left(u_{3}\right)+\ldots+d\left(u_{r+1}\right)-(r+1) r .
$$

However, since every vertex in $B_{i}$ is connected to exactly $i$ vertices in $A$, therefore

$$
e\left(G\left[A, B_{i}\right]\right)=i b_{i} .
$$




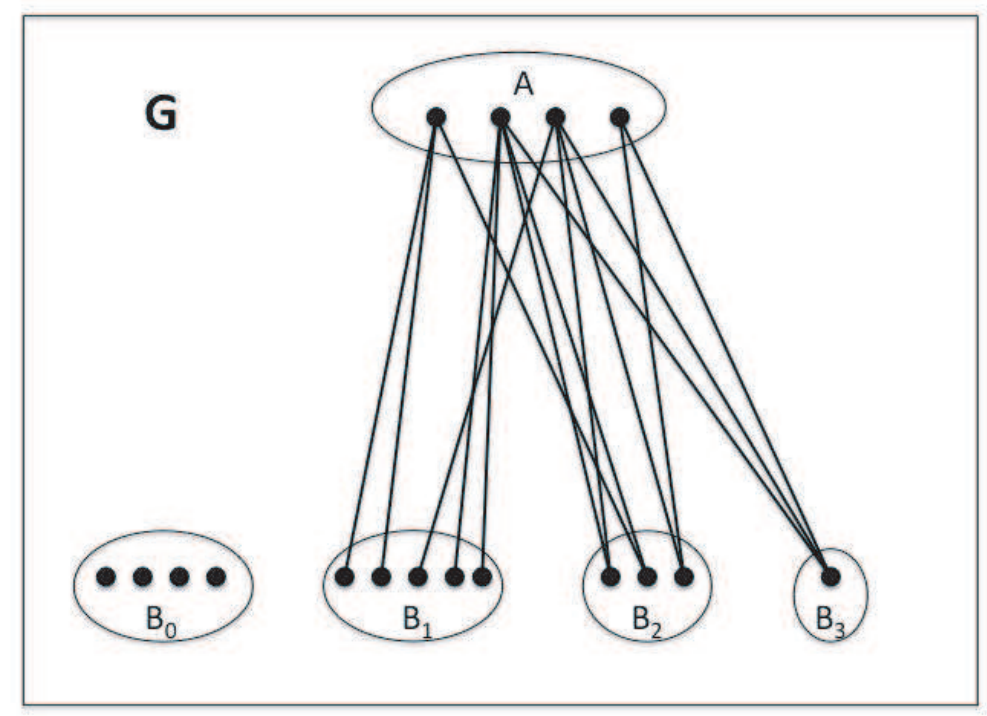

Figure 1: An example graph of $G[V(G)-A, A]$ in the $r=3, \lambda=4$ case.

As sets $B_{i}$ are pairwise disjoint,

$$
e(G[A, V(G)-A])=e\left(G\left[A, B_{0}\right]\right)+e\left(G\left[A, B_{1}\right]\right)+\ldots+e\left(G\left[A, B_{r}\right]\right),
$$

and since

$$
d\left(u_{1}\right)+d\left(u_{2}\right)+d\left(u_{3}\right)+\ldots+d\left(u_{r+1}\right) \geq(r+1)((r-1) k+s),
$$

we obtain that:

$$
\sum_{i=0}^{r} i b_{i} \geq(r+1)((r-1) k+s)-(r+1) r .
$$

In addition, since there are $n-(r+1)=r k+s-(r+1)$ vertices in $V(G)-A$, and $B_{0}, B_{1}, \ldots, B_{r}$ are all disjoint sets, we have that

$$
\sum_{i=0}^{r} b_{i}=r k+s-(r+1) .
$$

Now, by multiplying (2) by $r$ and subtracting it from (1), we obtain

$$
\sum_{i=0}^{r}\left(i b_{i}-r b_{i}\right) \geq(r+1)((r-1) k+s)-r(r+1)-\left(r^{2} k+r s-r(r+1)\right)=s-k .
$$

Hence,

$$
\sum_{i=0}^{r}(i-r) b_{i}=\sum_{i=0}^{r-1}(i-r) b_{i} \geq s-k
$$


which gives

$$
b_{r-1} \leq k-s-\sum_{i=0}^{r-2}(r-i) b_{i} .
$$

Consider an $(r+1)$-coloring of $G$. Since all vertices of $A$ are assigned distinct colors, and since every vertex in $B_{i}$ is adjacent to $i$ vertices of $A$, there are at most $(r+1)-i$ ways to color each vertex in $B_{i}$. As there are $(r+1)$ ! ways to color $A$, using $(3)$, we have that

$$
\begin{aligned}
P_{G}(r+1) & \leq(r+1) ! \prod_{i=0}^{r}(r+1-i)^{b_{i}} \\
& \leq(r+1) ! 2^{b_{r-1}} \prod_{i=0}^{r-2}(r+1-i)^{b_{i}} \\
& \leq(r+1) ! 2^{k-s} \prod_{i=0}^{r-2} \frac{(r+1-i)^{b_{i}}}{2^{(r-i) b_{i}}} \\
& \leq(r+1) ! 2^{k-s} \prod_{i=0}^{r-2}\left(\frac{r+1-i}{2^{r-i}}\right)^{b_{i}} \\
& \leq(r+1) ! 2^{k-s} .
\end{aligned}
$$

As $n=r k+s>r, k=\left\lfloor\frac{n}{k}\right\rfloor \geq 1$, and $0 \leq s<r$, we have either $s \geq 1$, or $k \geq 2$.

Suppose that $s \geq 1$. Then, as $r \geq 2$, we have

$$
\begin{aligned}
P_{T_{r}(n)}(r+1)-(r+1) ! 2^{k-s} & =(r+1) !\left(s 2^{k}+(r-s) 2^{k-1}-(r-1)-2^{k-s}\right) \\
& \geq(r+1) !\left(2^{k}+(r-1) 2^{k-1}-(r-1)-2^{k-1}\right) \\
& =(r+1) !\left(2^{k}+(r-2) 2^{k-1}-(r-1)\right) \\
& >0
\end{aligned}
$$

Note that this extends the result in [6] for $\lambda=3$ from $\left(2 k, k^{2}\right)$-graphs to $(2 k+1, k(k+1))$-graphs, and, hence, proves Theorem 1 for $r=2, \lambda=r+1=3$ case.

Finally we assume that $r \geq 3$ and $s=0$. If $k \geq 2$, we obtain

$$
\begin{aligned}
P_{T_{r}(n)}(r+1)-(r+1) ! 2^{k-s} & =(r+1) !\left(s 2^{k}+(r-s) 2^{k-1}-(r-1)-2^{k-s}\right) \\
& =(r+1) !\left(r 2^{k-1}-(r-1)-2^{k}\right) \\
& =(r+1) !\left((r-2) 2^{k-1}-(r-1)\right) \\
& \geq(r+1) !(2(r-2)-(r-1)) \\
& =(r+1) !(r-3) \\
& \geq 0
\end{aligned}
$$

with equality if and only if $k=2, r=3$, and $s=0$. This implies $n=2 \cdot 3+0=6$. 
Therefore we assume that $r=3$ and $n=6$. In this case, $A=\left\{u_{1}, \ldots, u_{4}\right\}$, and $P_{T_{r}(n)}(r+1)=P_{T_{3}(6)}(4)=4 !\left(0 \cdot 2^{2}+3 \cdot 2^{1}-2\right)=96$. Now, since $G$ has only 6 vertices, then $|V(G)-A|=2$, and $e(G[V(G)-A]) \leq e\left(K_{2}\right)=1$. In addition,

$$
e(G)=t_{3}(6)=12=e(G[A])+e(G[V(G)-A])+e(G[A, V(G)-A]) .
$$

Therefore we have

$$
12 \leq 6+1+\left(d\left(u_{1}\right)+d\left(u_{2}\right)+d\left(u_{3}\right)+d\left(u_{4}\right)-12\right),
$$

which leads to

$$
d\left(u_{1}\right)+d\left(u_{2}\right)+d\left(u_{3}\right)+d\left(u_{4}\right) \geq 17 .
$$

So, $e(G[A, V(G)-A]) \geq 5$. Let $V(G)-A=\{x, y\}, d_{A}(x) \geq d_{A}(y)$. Then $d_{A}(x)+$ $d_{A}(y) \geq 5$, and so $d_{A}(x) \geq 3$. If $d_{A}(x) \geq 4$, then $G$ contains a copy of $K_{5}$. This implies $P_{G}(4)=0<P_{T_{3}(6)}(4)$.

If $d_{A}(x)=3$, then $d_{A}(y)=2$. Now, there exist 4 ! ways to color properly the vertices in $A$. Each such coloring can be extended to a proper coloring of $G$ in at most two ways, as $x$ can be colored uniquely, and $y$ can be colored in at most 2 ways. This shows that $P_{G}(4) \leq 4 ! \cdot 2=48$, which is less than $P_{T_{r}(n)}(r+1)=P_{T_{3}(6)}(4)=96$, and so this ends the proof of Case 2, and of Theorem 1.

\section{Acknowledgment}

The authors are thankful to Sebastian Cioabă, Gary Ebert, and Qing Xiang for their comments on the original version of our proof. We are thankful to David Galvin for bringing to our attention the preprint [16], and to Serguei Norine for sharing with us his preprint [9]. Finally, we are thankful to the anonymous referee for several useful remarks.

\section{References}

[1] E. Bender and H. Wilf. A theoretical analysis of backtracking in the graph coloring problem. J. Algorithms 6 (1985). 275-282.

[2] G. Birkhoff. A determinant formula for the number of ways of coloring a map. Annals of Mathematics 14 (1912). 4246.

[3] B. Bollobás. Modern Graph Theory Springer-Verlag. Berlin. 1998.

[4] F. Lazebnik. On the greatest number of 2 and 3 colorings of a $(v, e)$-graph. J. Graph Theory 13 (1989). 203-214.

[5] F. Lazebnik. Some corollaries of a theorem of Whitney on the chromatic polynomial. Discrete Math. 87 (1991). 53-64. 
[6] F. Lazebnik, O. Pikhurko, and A. Woldar. Maximum number of colorings of $\left(2 k, k^{2}\right)$ graphs. J. Graph Theory 56 (2007). 135-148.

[7] N. Linial. Legal coloring of graphs. Combinatorica 6 (1986). 49-54.

[8] P-S. Loh, O. Pikhurko, and B. Sudakov. Maximizing the number of $q$-colorings. Proc. London Math. Soc. 101 (2010). 655-696.

[9] S. Norine. Turán Graphs and the Number of Colorings. SIAM J. Discrete Math., to appear. http://www. math.princeton. edu/ snorin/papers/TuranMaxColor.pdf.

[10] R.C. Read. An introduction to chromatic polynomials. J. Combinatorial Theory 4 (1968). 52-71.

[11] R.C. Read and W.T. Tutte. Chromatic polynomials. In: (2nd ed.), Selected Topics in Graph Theory 3, Academic Press, New York (1988). 15-42.

[12] R.C. Read and G.F. Royle. Chromatic roots of families of graphs. Graph theory, combinatorics, and applications. Vol. 2 (Kalamazoo, MI, 1991), 1009-1029, WileyIntersci. Publ., Wiley, New York, 1991.

[13] S.N. Tofts. Number of Colorings of $r$-Partite Turán Graphs. Master's thesis. University of Delaware. 2010.

[14] P. Turán. On an extremal problem in graph theory (in Hungarian). Mat. Fiz. Lapok, 48:436-452, 1941.

[15] H. Wilf. Backtrack: an $O(1)$ expected time algorithm for the graph coloring problem. Information Processing Letters 18 (1984). 119-121.

[16] Y. Zhao. The Bipartite Swapping Trick on Graph Homomorphisms, preprint. http://web.mit.edu/yufeiz/www/bipartite_swapping_trick.pdf. 